(C) 2019 IEEE. Personal use of this material is permitted. Permission from IEEE must be obtained for all other uses, in any current or future media, including reprinting/republishing this material for advertising or promotional purposes, creating new collective works, for resale or redistribution to servers or lists, or reuse of any copyrighted component of this work in other works. 


\title{
Underwater Archaeology with Light AUVs
}

\author{
Maria Costa ${ }^{1}$, José Pinto ${ }^{1}$, Manuel Ribeiro ${ }^{1}$, Keila Lima ${ }^{1}$, Alexandre Monteiro ${ }^{2}$, Peter Kowalczyk ${ }^{3}$, João Sousa ${ }^{1}$ \\ mariacosta@fe.up.pt, almonteiro@fcsh.unl.pt, peter.kowalczyk@oceanfloorgeophysics.com
}

\begin{abstract}
For millennia, ships were lost at sea leading to the loss of an estimated 3 million vessels. This paper describes a light autonomous underwater vehicle specially tailored for coastal archaeology applications where a big part of this heritage sites should be found. This specific vehicle includes sonars, an optical camera and a magnetometer to detect and identify archaeological artifacts in the ocean bottom or underneath. It can be used isolated or as part of a team of AUVs for faster surveys. We describe the hardware, its simplified operation using custommade software and overview some results in different areas, where these vehicles have been used to detect several wrecks and other important artifacts.
\end{abstract}

Index Terms-Autonomous Underwater Vehicle, Underwater Archaeology

\section{INTRODUCTION}

Before there were farmers, there were sailors. For millennia people crossed rivers, lakes, seas, and later, even oceans. From shallow wading creeks to coast hugging navigation, raw materials, manufactured goods, ideas, ideologies, religion, even genes and diseases disseminated via waterways, the safest, less costly and sometimes faster routes between human communities, production centers, bulk trade markets and consumers. Also, for millennia, ships were lost at sea. Poor navigation skills, storms, acts of war, negligence, piracy or just sheer bad luck were responsible for the loss of an estimated 3 million vessels, the remains of which compose humankind's Underwater Cultural Heritage (UCH). Of these projected 3 million archaeological sites, only a fraction has been located and investigated. As shipwrecks deposited on the seabed are non-renewable sources to understanding and knowledge of our past, providing the necessary socio-historic component to ocean understanding, illustrating the history not only of humanity's relations with the oceans, but also of climate change [1], the scientific community is working against the clock to secure as much of this heritage as possible for posterity.

As current methods for searching underwater archaeological sites involve many steps with expensive equipment and timeconsuming analysis and as climate change, treasure hunting and increased commercial activities in the marine environments have put this cultural heritage under pressure [2], the need for new and efficient methods for recording and managing underwater cultural heritage is now paramount and urgent [3].

\footnotetext{
${ }^{1}$ Laboratório de Sistemas e Tecnologia Subaquática, Faculdade de Engenharia da Universidade do Porto

2 Instituto de Arqueologia e Paleociências, Faculdade de Ciências Sociais e Humanas, Universidade Nova de Lisboa

${ }^{3}$ Ocean Floor Geophysics
}

As surveys expands further into the ocean depths, AUVs will play a larger part in nautical archaeology, by automating the process of searching for underwater archaeological sites, in a safer, more economical and much more efficient way.

\section{A. State of the art}

Archaeological surveys vary in respect to prior knowledge of artifacts that are being looked for. When there exist prior reports or documentation about known wrecks, a typical first step involves performing large side scan surveys to detect any man-made structures that are above the ocean surface. For this, large oceanographic ships or small vessels towing a sonar are typically employed. As second step, if contacts are detect by sonars, divers or small ROVs are used to photograph the approximate locations of the contacts. In case the wreck is buried underneath the ocean floor, large sub-bottom profilers (on large ships) can be used to detect changes in the density of the ocean floor or, alternatively, magnetometers can be used to detect anomalies that can be correlated with sunken objects.

When compared to light AUVs, large ships are very expensive to operate and they are not applicable to coastal waters as they typically cannot steam that close to shore (being that the reason why many wrecks actually happened). Sensors towed by small vessels, on the other hand, are cost-efficient but results are worse in that it is not possible to maintain a constant distance between the sensor and the bottom while also maintaining the speed of the vessel. Finally, surveys requiring divers are very expensive and not as efficient as the divers localization is poor which makes it difficult to perform any systematic surveys.

Using AUVs to perform such surveys is a cost effective solution which yields higher spatial resolutions, with less positioning errors - more precise path following, less interference from motion-induced noise, etc. AUVs are more suitable to detect small targets and stand as a much more reliable and systematic solution for shallow/coastal water surveys, where vessel-based expeditions have difficulties to operate and have more erratic trajectories and diver surveys are labor - and cost - intensive, requiring highly trained professionals.

In terms of sensing payloads, the most common sensors being used for archaeology in AUVs are sidescan and multibeam sonars, as well as towed magnetometers and subbottom profilers. While sonars are very effective to cover large areas quickly, the magnetometers and sub-bottom profilers can detect artifacts buried underneath the ocean floor. The magnetometers can be mounted on the AUV directly, requiring the AUV to be purpose built to be magnetically quiet. This necessitates a specialized and expensive AUV not suited to 
other AUV missions. The second method is to tow the magnetometer behind the AUV. This is intuitively a simple approach and allows a standard magnetometer to be used. However, a towed magnetometer complicates launch and recovery of the AUV, degrades the AUV performance, and increases the risk of losing the AUV due to entanglement of the magnetometer in a ship propeller.

The integration of these sensors on small AUVs is still quite challenging, mainly due to AUV's limited size and space for payloads. The challenge we sought out to address in this work was to assess the capability of light AUVs for marine archaeology with present-day sensors, towards a solution significantly more affordable and of simpler operation. The light AUVs used in our approach don't require any special equipment for launch and recovery or other expensive logistics. By using self-compensated sensors instead of towing them, these AUVs can be deployed from a small boat or from shore, which furthermore reduces the costs of operations.

Similar work to what we are proposing in this document can be found in [4], where near shore surveys are done using an AUV equipped with a sidescan sonar and a towed magnetometer. In [5] work regarding multi-AUV collaboration for marine archaeology is presented and in [6] a summary of use cases of robotic technologies for underwater archaeology is reported, where archaeologists requirements were identified for all the phases of a campaign by the proposed methodology.

\section{TOOLS AND TECHNOLOGIES}

The main innovations used in our work for simplified archaeological surveys in coastal areas are man-portable and re-configurable AUVs, as well as simplified operations through in-house developed software, together with self-compensating magnetometers from Ocean Floor Geophysics. These tools and technologies are a good starting point towards answering the question we propose to address in this work.

\section{A. The LAUV - Light Autonomous Underwater Vehicle}

The Underwater Systems and Technology Laboratory from Porto University (LSTS) has been developing and operating light AUVs for different scientific applications in coastal and open sea waters for the last decade, resulting in the creation of a Spin-off company (OceanScan Marine Systems and Technology ${ }^{1}$ ) and several vehicles being sold worldwide. The LAUV [7] is a one-man portable AUV that requires a minimal operational setup and can be designed to fit different sensing payloads and autonomous behaviors. Bottom-mapping AUVs stay submerged for most of the time and, as such, these systems must fuse data from multiple sensors for precise underwater localization.

1) Localization: In the case of LAUV vehicles used by LSTS, they use a GPS (at surface) and IMU / DVL / AHRS sensors when submerged. Moreover, the LAUV vehicles can also use acoustic positioning (underwater GPS) by defining apriori the positions of carefully placed acoustic beacons that

\footnotetext{
${ }^{1}$ https://www.oceanscan-mst.com/
}

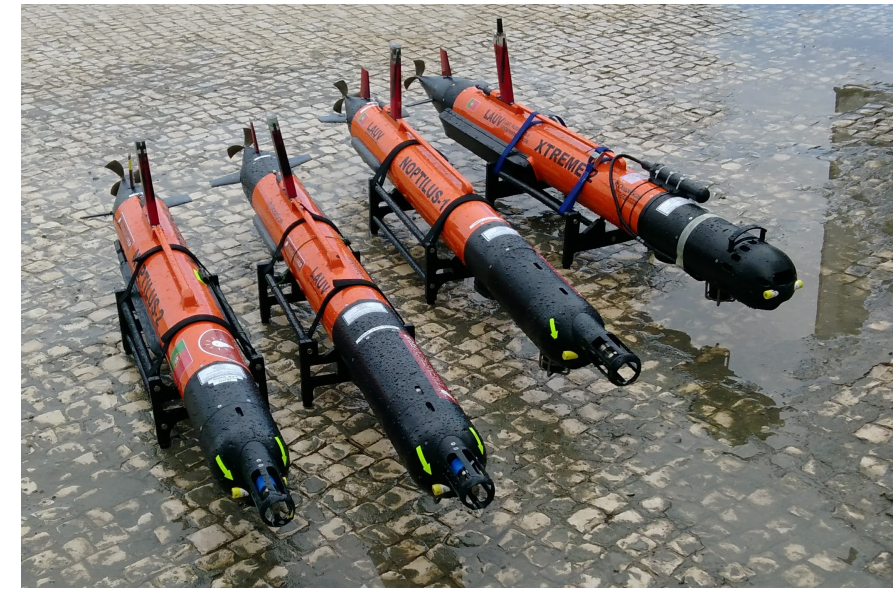

Fig. 1. Bottom-mapping LAUV vehicles. The rightmost AUV (LAUVXtreme-2) is equipped with Sidescan sonar, video camera and selfcompensating magnetometer.

stay static throughout the survey. Alternatively, other vehicles can also be used as acoustic beacons, using a station keeping autonomous behavior to maintain its position.

2) Communication and Emergency Systems: The LAUV vehicles can be controlled using just Wi-Fi (when they come to the surface). Moreover, if they emerge far away from the base station (no Wi-Fi link), GSM can be used to obtain the state and send commands to the vehicles as well as Iridium satellite communications. When the vehicles submerge, they can still communicate with a base station by using an acoustic modem. This is very important in areas with ship traffic, as the operator can interrupt or change the plan the vehicle is executing in case of incoming vessels.

3) Sensing Payloads: The bottom-maping vehicles used by LSTS have different sets of payloads. All vehicles carry a sidescan sonar (Imagenex, Klein or Edgetech). The different sonars vary mostly in the resolution they provide, being possible to locate smaller objects faster with high-frequency sonars. Some of the vehicles also carry a video camera which can be used to identify previously detected contacts. In comparison with sonars, cameras provide even higher resolutions but only capture a narrow area of the bottom, it is more dependent on sea state conditions and requires the vehicle to travel really close to the bottom which can increases the probability of the vehicle becoming trapped in fishing nets. Finally, a recent adition has been adding a magnetometer to these vehicles, described next.

\section{B. Self-Compensating Magnetometer}

An Ocean Floor Geophysics self compensated magnetometer (SCM) was used for this work. The magnetometer mounts directly on the AUV, and after a proprietary compensation maneuver is executed, provides magnetometer data compensated for the AUV heading errors.

When using LAUV with the SCM for magnetometer surveys, a survey pattern can be designed and executed in one dive 


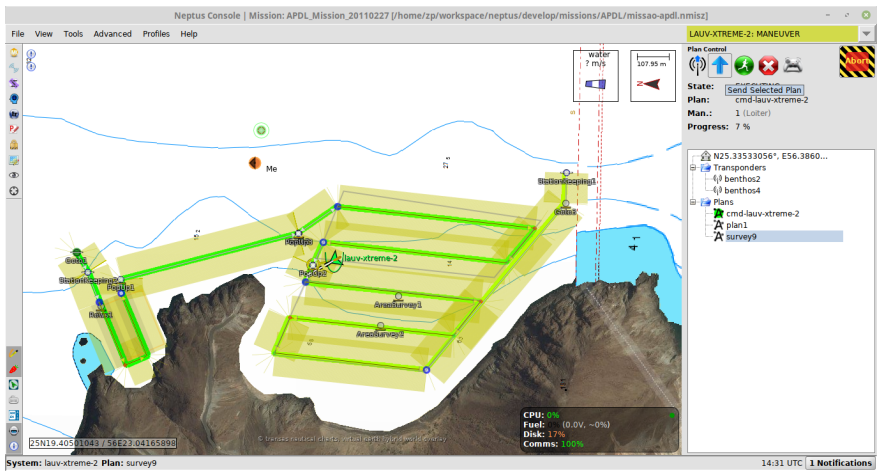

Fig. 2. Neptus Operator Console being used to define and simulate a plan prior to execution.

to search for magnetic objects on or below the seafloor. As the AUV can travel close and at constant distance to the seafloor, magnetic anomalies are stronger than they would be measured close to the sea surface. The magnetic data is intrinsically co-registered with the LAUV side-scan sonar, as both are collected at the same time at high resolution. The navigational accuracy of the LAUV survey allows both profile and contour analysis of the magnetic data to be done. When planning to map a previously known magnetic anomaly, one should make several well controlled passes across it, to provide more information about the source of the anomaly, not available when doing only a single transect. The same AUV can be used in a prospecting mode with single or widely spaced traverses covering a wide swath with the side-scan sonar to investigate larger areas. Any targets identified in the sonar image can be subsequently investigated in detail.

The interpretation of AUV magnetic data uses methods well established in the mineral and oil exploration industry, and in land based archaeological work [8]. In an AUV survey, many anomalies come from iron objects in the seafloor, which are strongly magnetized and are likely to have strong remanent magnetization not typical in geologic sources seen in land based surveys. A rapid method of presenting such magnetic anomalies is to compute the analytic signal amplitude [9], which illustrates the total magnetic gradient and is insensitive to the direction of magnetization of the source or the direction of the ambient magnetic field. To interpret these anomalies in 3-D, a first approach and rapid method is to use the Analytic Signal Vertically Integrated (ASVI) [10] and 3D voxel interpretation [7].

\section{Software Tools}

With the evolution of the LAUV as a reliable platform for diverse surveying applications, the software toolchain developed by LSTS to support these systems has also evolved throughout the years. The LSTS Toolchain is an open-source software suite tailored for mixed-initiative control of networked heterogeneous unmanned systems [11]. The elements of the toolchain more relevant for this work are the following:

- Neptus Operator Console: This application allows the operators to choose the widgets and map layers used to visualize the data coming from one or more connected vehicles. The vehicles can be of different types (AUVs, ROVs ASVs and UAVs) and their data is easily correlated. Moreover, if more than one consoles are connected to the same network, they can also share information such as points of interest and designed autonomous plans. Design of autonomous missions is simplified by using plan generators that account for all vehicle and sensor limitations, making sure a user-defined area is mapped completely. Moreover, before execution the generated plans can be visualized and simulated to check for potential risks and informed validation by the operators (see figure 2).

- Neptus Mission Review and Analysis: This application, also part of the Neptus software framework, allows revising data previously acquired by the autonomous vehicles. Irrespective of the sensors used, all data can be geo-referenced and overlayed on a map right after it is downloaded from the vehicle(s). Examples of such displays are presented next, in section III.

- DUNE: Stands for DUNE Unified Navigation Environment and is the onboard software running on the vehicles. Handles control, navigation, simulation, networking, sensing, and actuation. It's very portable (small footprint, CPU architecture and OS independent) and it's adaptable to very diverse hardware systems. The fact that all vehicles (and communication gateways) are running the same software, simplifies operation as the command set is shared and is much simpler to combine data acquired by multiple vehicles. DUNE also supports many different kinds of sensor payloads (sonars, environmental probes, magnetometers, cameras, etc) making its use normalized across systems.

\section{Methodology}

We present a methodology to survey a coastal area in search for archaeological artifacts using one or more man-portable AUVs. These AUVs have simplified operation and can be deployed from shore or from a small vessel.

The methodology is divided into several phases: sparse surface survey, contact identification and sub-surface survey.

For this work, we propose a LAUV configuration similar to the one used in LAUV-Xtreme-2 (see figure 3), equipped as follows:

- Ocean Floor Geophysics Self-Compensated Magnetometer

- Klein 3500 High-Resolution Side Scan Sonar (with Bathymetry option)

- Point Grey Camera - Chameleon 1.3 MP Color USB 2.0 (Sony ICX445)

Our software toolchain allows us to plan the missions on-the-fly, as well as processing all the collected data in a correlated manner, right after the mission is performed, which is quite useful and an improvement when comparing to the conventional setups. Still, for this application, we decided to create a dedicated plugin for Neptus to improve the ability 


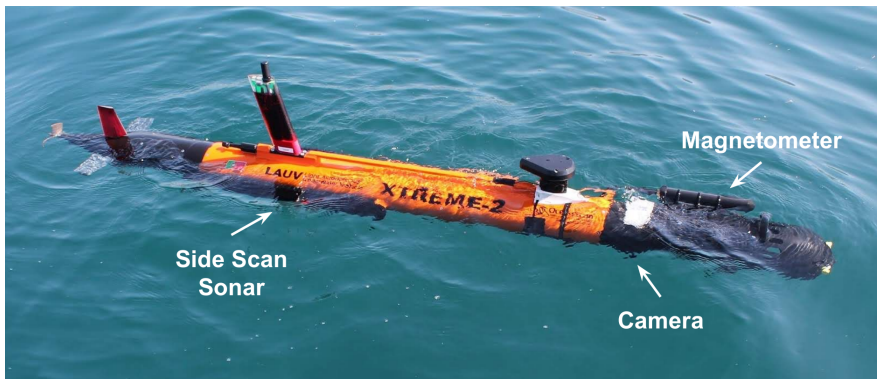

Fig. 3. LAUV-Xtreme-2 AUV payload configuration.

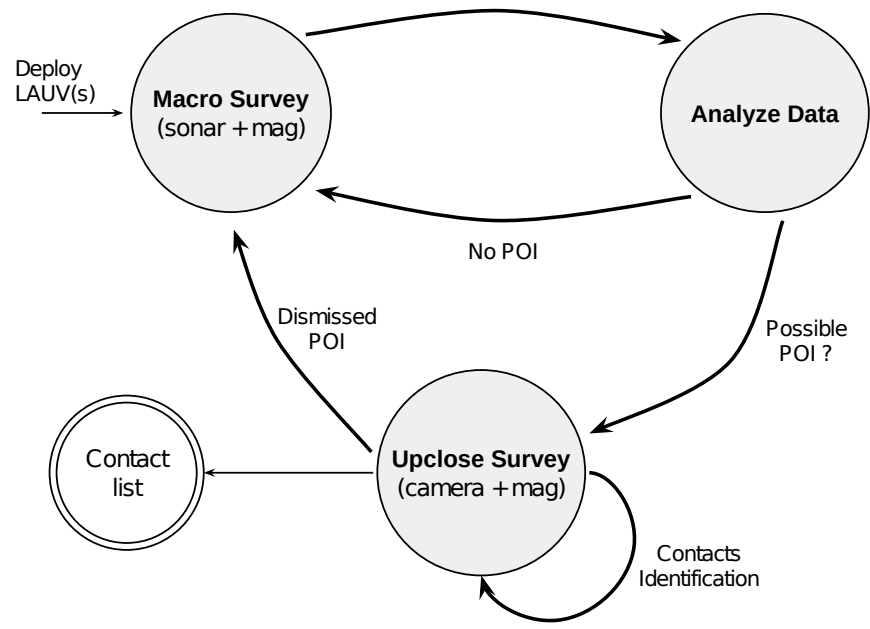

Fig. 4. Proposed methodology with different survey and revision phases.

to generate survey plans for one or multiple vehicles more quickly (where the operator just needs to select the desired area, the desired vehicles and the desired payloads to use). This turned out to be quite valuable when in challenging environments.

Finally, the proposed operational workflow is the following:

1) Define the area of interest in the map and generate a plan (or plans) to survey the area using sidescan sonar and magnetometer;

2) Review data and identify possible contacts and points of interest;

3) Create close-up surveys with magnetometer and camera over detected points of interest.

4) Revise the data and decide based on camera and magnetometer footprints, if the contacts are of archaeological value.

5) Compile a list of archaeological artifacts with location and sonar / optical / magnetometer footprints.

Note that the base station can be either at the shore (if the target area is close by) or on a small support surface vessel. A vessel can be used to move faster to the deployment location and then stays stationary (anchored) for the duration of the surveys to monitor the execution. In parallel with this, more vehicles can be deployed for faster surveys.

This methodology requires one (or more) specialized opera-

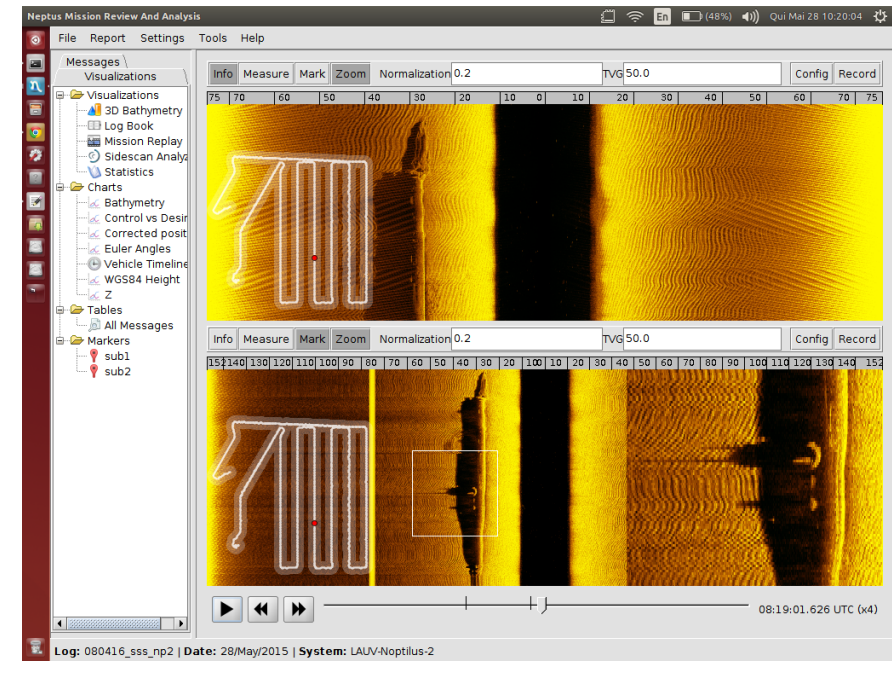

Fig. 5. Neptus MRA being used to inspect sidescan data from U1277 survey.

tor(s) that is capable of identifying potential contacts in sidescan sonar. This results in either transmitting large quantities of information to shore, where the specialist resides or having the specialist onboard to look at the data as it becomes available. Again, if using multiple vehicles a single specialist can be used for inspecting the data of all vehicles, lowering costs. Finally, an archaeologist is required to look at the identified contacts and decide whether each is of archaeological value.

\section{Use CASES AND Results}

The methodology in section III has been employed multiple times both for demonstration and archaeology purposes. Next we describe objectives and results of different field deployments.

\section{A. U-1277 Submarine survey}

The main diving spot which is close to Porto University is the wreck of U-1277, a German U-boat from World War II. This submarine is sunk off the coast of Matosinhos at a depth of 27 meters. This area is well known by divers but good visibility days are very rare due to great energy and resulting turbidity. This was done with the help of a local diving group and the Portuguese Navy, as part of a bigger exercise.

At this time, the main objective for LSTS was to demonstrate the use of sidescan sonars for archaeology applications. This site was deemed to be spot-on for this purpose, since it is a well known target that still remains quite intact and seating on the seabed. All specifications of the sunken vessel can be easily obtained, which can be used to compare with the acquired images. For this survey we used two of our LAUVs, both carrying sidescan sonar and carrying also multibeam sonar and a camera. The resulting sidescan images allows measuring and also identifying different chimneys and structures on the wreck (see figure 5). 


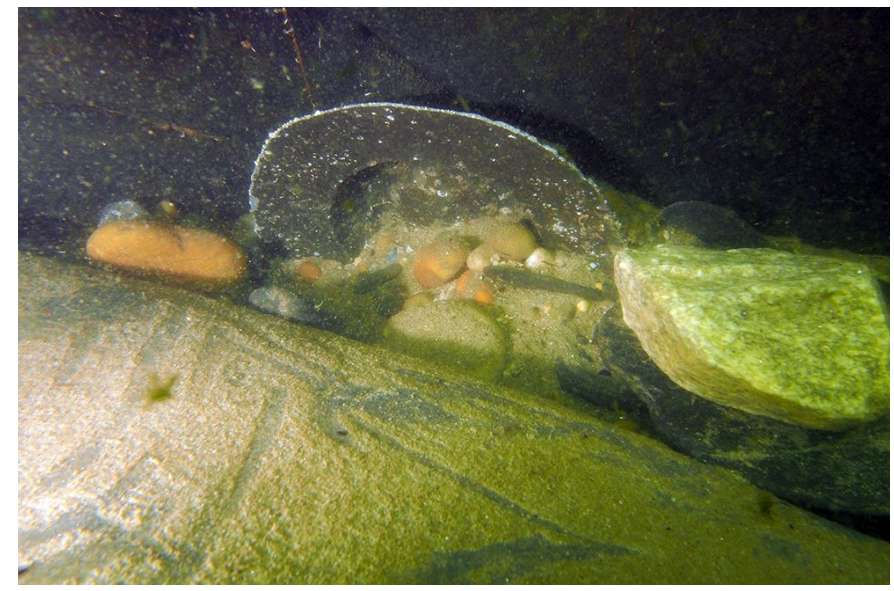

Fig. 6. Artifacts from Belinho shipwreck with dating estimated from XVII century (C) John Sexton.

\section{B. Esposende Surveys}

In 2017 we started working together with OceanScan-MST and Ocean Floor Geophysics (OFG) to test and evaluate the behaviour of their self-compensating magnetometer with our LAUV. Moreover, recently a set of artifacts came ashore in Belinho, a beach close to Esposende in the north of Portugal, following heavy storms. The artifacts consisted of zinc plates, candle-holders and cannon balls. Archaeologists contacted LSTS to deploy an AUV in the area to try locating the source of such debris. Two surveys were conducted, the first taking place in April 2017 and second on August 2017.

In April 2017 the objective was quite simply to survey the coast close to the area where the artifacts were found to try to detect any man-made structures and otherwise any magnetic anomalies. Several surveys were conducted both at the surface and close to the bottom, mapping the coast up to the beach and using a sidescan swath width of 75 meters. Even tough the support vessel was of small size, it could not go where the AUV was conducting most of its surveys with 3-5 meters of depth and waves.

The area is very rocky and rugged, which didn't help with the interpretation of sidescan results. However, the magnetometer data could be used to detect several anomalies in the area, most still to be inspected by archaeologists. In one of those anomalies, zinc plates and canons were also found in between the rocks.

\section{Sharjah}

Recently, in November of 2018, the Sharjah Archaeological Authority invited a group of Portuguese archaeologists to conduct surveys on the coast of this Emirate. An extensive survey off Khor Fakkan was made using two AUVs using the presented methodology. Moreover, as the team included underwater archaeologists, some of the detected contacts were inspected during the expedition. The purpose of this field mission was to demonstrate the capabilities of the proposed approach, as well as identifying potential sites of interest in

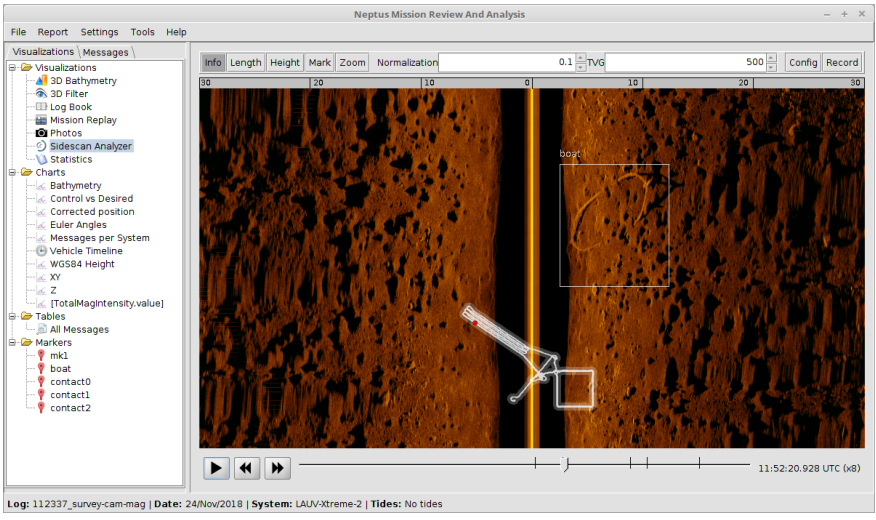

Fig. 7. Sidescan contact where a vessel frame can be easily spotted.

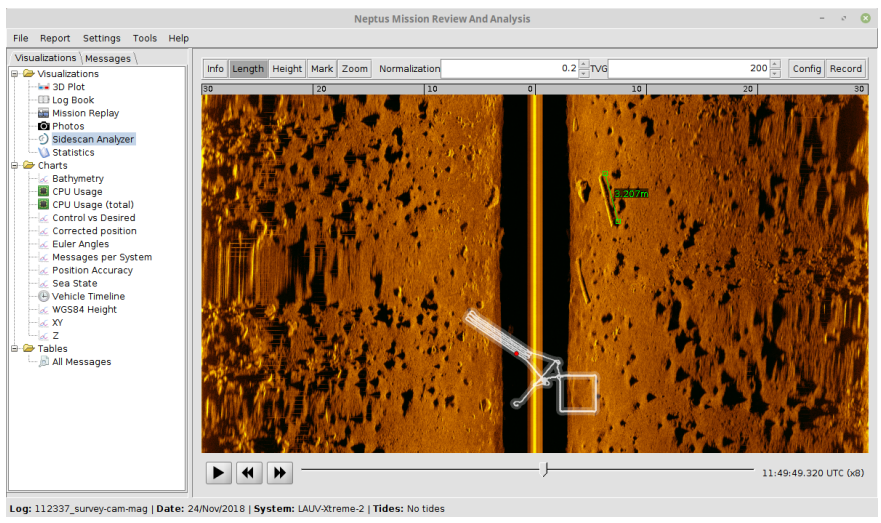

Fig. 8. Another anomaly that could be identified both in the sidescan and magnetometer data.

the area, known to have had an old Portuguese fort in the 16th century.

In the first day of operations the contacts in figures 7 and 8 were detected. While the first was undoubtedly a boat (or part of it), the second could be anything from a tree to a canon. A second survey was conducted with camera and magnetometer to better identify the contacts. Both corresponded to magnetic anomalies (see figure 9). Since the survey crossed the location of the boat several times, there were several peaks visible in the magnetometer data.

A large amount of the coast was covered using sidescan at the rate of one squared kilometer per hour. Inspection of these data indicated the presence of several anomalies in the bottom, mostly due to its proximity to a large harbor. Some of the contacts were later selected for identification using camera and magnetometer. A total of 7 wrecks were found, 5 of them identified using camera or magnetometer. The archaeologists dove in several of the located sites for more thorough inspections. Finally, a quad copter UAV was also used to augment the collected data with 3D reconstructions of the shoreline. 


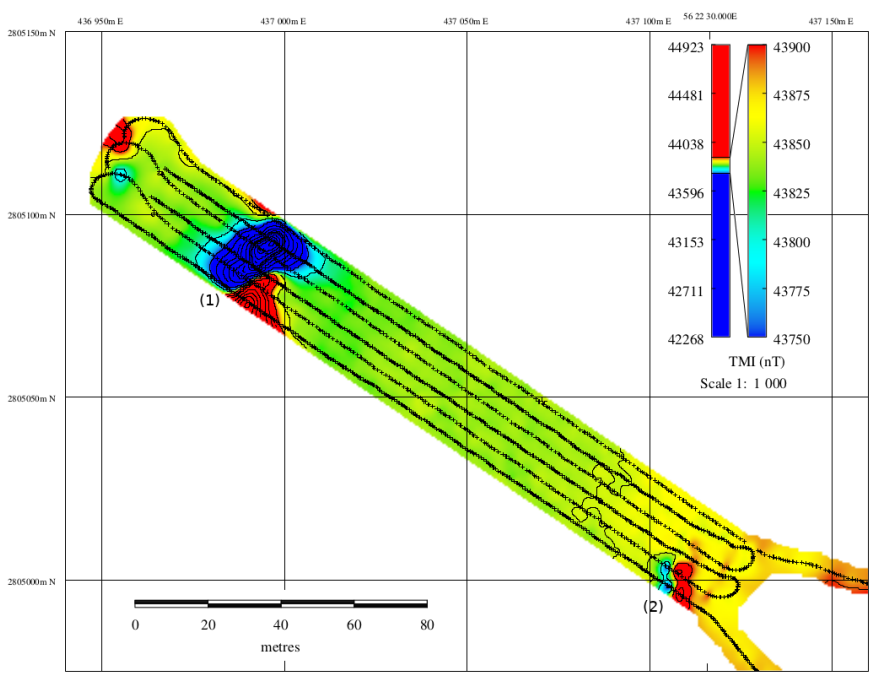

Fig. 9. Magnetometer survey where two main anomalies are prominent, the ones in figures 7 and 8 . Anomaly (1) corresponds to the vessel in figure 7 while anomaly (2) corresponds to the long shaped objects in figure 8 .

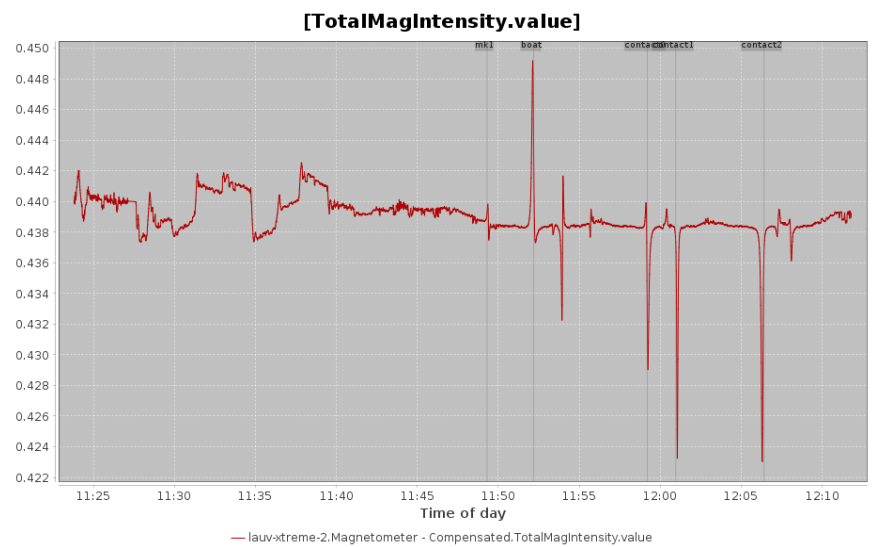

Fig. 10. Total magnetic intensity plotted against time. The vertical lines represent locations where features of interest were detected in the sidescan, which corresponded to the peaks (anomalies) in total magnetic intensity.

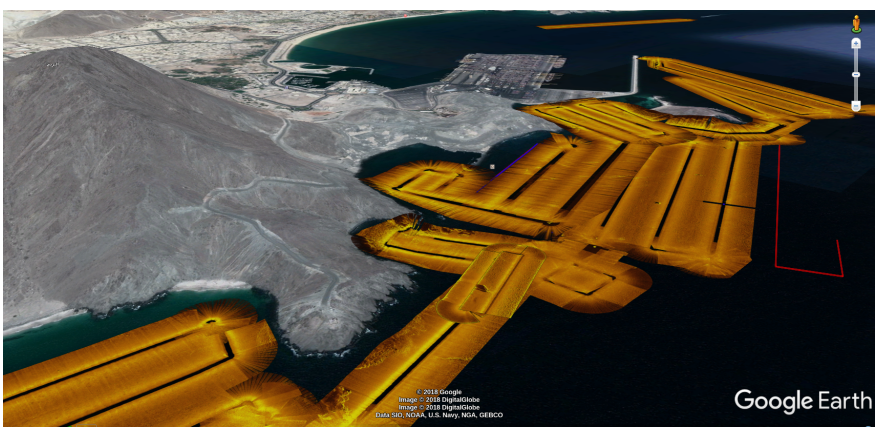

Fig. 11. Sidescan surveys conducted off Sharjah overlayed on a map where Shark island and Khor Fakkan's harbor are also present.

\section{CONCLUSIONS}

We have presented a cost-efficient approach to conduct archaeology in coastal waters. The data that can be acquired with AUVs is of much greater quality than the ones obtained with ships or divers due to the better positioning and maneuverability of the AUVs. The propose AUV configuration proved to be very effective to detect and identify different previously unknown wrecks.

Even though a specialist is still required to interpret the data acquired by the AUVs, s/he can revise the data coming from multiple AUVs making her more efficient. The data that was obtained was of high quality and sufficient resolution for underwater archaeology.

This approach will be further employed in the field within the scope of a new project to survey shipwrecks in South Portugal $^{2}$.

\section{ACKNOWLEDGMENTS}

This work was partially funded by the European Union's Horizon 2020 research and innovation programme under grant agreement No 731103 and MARINFO - Integrated Platform for Marine Data Acquisition and Analysis, supported by Norte Portugal Regional Operational Programme (NORTE 2020), under the PORTUGAL 2020 Partnership Agreement, through the European Regional Development Fund (ERDF). The authors would also like to acknowledge all the support provided by the Esposende municipality and Sharjah Archaeology Authority for the surveys off Esposende and UAE, respectively, as well as the Portuguese Navy.

\section{REFERENCES}

[1] J. Sopova, "Submerged memory," UNESCO Courier, vol. 1, 2009.

[2] S. M. Nornes, M. Ludvigsen, Øyvind Ødegard, and A. J. SØrensen, "Underwater photogrammetric mapping of an intact standing steel wreck with rov," IFAC-PapersOnLine, vol. 48, no. 2, pp. 206 - 211, 2015, 4th IFAC Workshop onNavigation, Guidance and Controlof Underwater VehiclesNGCUV 2015.

[3] J. Rutledge, W. Yuan, J. Wu, S. Freed, A. Lewis, Z. Wood, T. Gambin, and C. Clark, "Intelligent shipwreck search using autonomous underwater vehicles," 05 2018, pp. 1-8.

[4] D. Hrvoic, "High-resolution near-shore geophysical survey using an autonomous underwater vehicle (auv) with integrated magnetometer and side-scan sonar,' Master's thesis, School of Graduate Studies, McMaster University, Hamilton, Ontario, 2014.

[5] N. Tsiogkas, G. Frost, N. Monni, and D. Lane, "Facilitating multi-auv collaboration for marine archaeology," in OCEANS 2015 - Genova, May 2015, pp. 1-4.

[6] B. Allotta, R. Costanzi, A. Ridolfi, O. Salvetti, M. Reggiannini, M. Kruusmaa, T. Salumae, D. M. Lane, G. Frost, N. Tsiogkas, M. Cocco, L. Gualdesi, G. Lacava, D. Roig, H. T. Gundogdu, M. I. C. Dede, S. Baines, S. Tusa, P. Latti, and D. Scaradozzi, "The ARROWS project: robotic technologies for underwater archaeology," IOP Conference Series: Materials Science and Engineering, vol. 364, p. 012088, jun 2018

[7] Y. Li and D. W. Oldenburg, "3-D inversion of magnetic data," Geophysics, vol. 61, no. 2, pp. 394-408, 04 1996. [Online]. Available: https://doi.org/10.1190/1.1443968

[8] C. Reeves, Aeromagnetic Surveys: Principles, Practice and Interpretation. Geosoft, 2005.

[9] I. Macleod, K. Jones, and T. F. Dai, "3-d analytic signal in the interpretation of total magnetic field data at low magnetic latitudes," Exploration Geophysics - EXPLOR GEOPHYS, vol. 24, 011993.

[10] J. Paine, M. Haederle, and M. Flis, "Using transformed tmi data to invert for remanently magnetised bodies," Exploration Geophysics - EXPLOR GEOPHYS, vol. 32, 012001.

[11] J. Pinto, P. S. Dias, R. Martins, J. Fortuna, E. Marques, and J. Sousa, "The lsts toolchain for networked vehicle systems," in 2013 MTS/IEEE OCEANS - Bergen, June 2013, pp. 1-9.

\footnotetext{
${ }^{2}$ https://www.mergulho-na-historia.pt/
} 